\title{
Lyapunov-type inequalities for $2 M$ th order equations under clamped-free boundary conditions
}

Kohtaro Watanabe ${ }^{1 *}$, Kazuo Takemura ${ }^{2}$, Yoshinori Kametaka ${ }^{3}$, Atsushi Nagai ${ }^{2}$ and Hiroyuki Yamagishi ${ }^{4}$

\footnotetext{
*Correspondence: wata@nda.ac.jp ${ }^{1}$ Department of Computer Science, National Defense Academy, 1-10-20 Hashirimizu, Yokosuka, 239-8686, Japan

Full list of author information is available at the end of the article
}

\begin{abstract}
This paper generalizes the well-known Lyapunov-type inequalities for second-order linear differential equations to certain $2 M$ th order linear differential equations

$$
(-1)^{M} u^{(2 M)}(x)-r(x) u(x)=0 \quad(-s \leq x \leq s)
$$

under clamped-free boundary conditions. The usage of the best constant of some kind of a Sobolev inequality helps clarify the process for obtaining the result.
\end{abstract}

\section{Introduction}

Let us consider the second-order linear differential equation

$$
\left\{\begin{array}{l}
u^{\prime \prime}(x)+r(x) u(x)=0 \quad(-s \leq x \leq s) \\
u( \pm s)=0
\end{array}\right.
$$

where $r \in C((-s, s), \mathbb{R})$. It is well known that the Lyapunov inequality

$$
\int_{-s}^{s} r^{+}(x) d x>\frac{2}{s}
$$

gives a necessary condition for the existence of non-trivial classical solutions of (1), where $r^{+}(x)=(r(x)+|r(x)|) / 2$. There are various extensions and applications for the above result; see, for example, surveys of Brown and Hinton [1] for relations to other fields and Tiryaki [2] for recent developments. Extensions to higher-order equations

$$
\left\{\begin{array}{l}
u^{(n)}(x)+r(x) u(x)=0 \quad(-s \leq x \leq s), \\
\text { Boundary Conditions, }
\end{array}\right.
$$

will be one important aspect. The first result for the high-order equation (3) is due to Levin [3], which states without proof:

\section{Springer}

๑ 2012 Watanabe et al.; licensee Springer. This is an Open Access article distributed under the terms of the Creative Commons Attribution License (http://creativecommons.org/licenses/by/2.0), which permits unrestricted use, distribution, and reproduction in any medium, provided the original work is properly cited. 
Theorem A Let $n=2 M$, and a non-trivial solution of (3) satisfies the clamped boundary condition, $u^{(i)}( \pm s)=0(i=0,1, \ldots, M-1)$. Then it holds that

$$
\int_{-s}^{s} r^{+}(x) d x>\frac{2^{2 M-1}(2 M-1)\{(M-1) !\}^{2}}{s^{2 M-1}} .
$$

Later, Das and Vatsala [4] gave the proof and extended the result by constructing the Green function. Other interesting developments for higher-order equations are seen in [5-9]. For example, as shown in Yang [8], Lyapunov-type inequalities can be obtained under the following conditions:

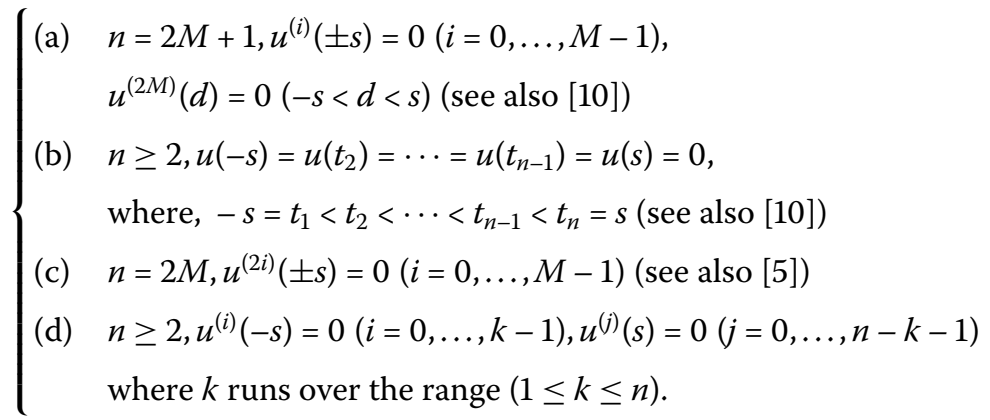

Here we note for the condition (c), very recently Çakmak [11], He and Tang [12], He and Zang [13] and [14] improved and extended the results of [5] and [8]. This paper considers the necessary condition for the existence of a non-trivial solution of the $2 M$ th order linear differential equation

$$
(-1)^{M} u^{(2 M)}(x)-r(x) u(x)=0 \quad(-s \leq x \leq s)
$$

under yet another boundary condition:

\section{Clamped-free boundary condition}

$$
u^{(i)}(-s)=0, \quad u^{(M+i)}(s)=0 \quad(i=0, \ldots, M-1) .
$$

The main result is as follows.

Theorem 1 Suppose a non-trivial solution u of (4) exists under the clamped-free boundary condition, then it holds

$$
\int_{-s}^{s} r^{+}(x) d x>\frac{\{(M-1) !\}^{2}(2 M-1)}{(2 s)^{2 M-1}} .
$$

Moreover, the estimate is sharp in the sense that there exists a function $r(x)$, and for this $r(x)$, the solution $u$ of (4) exits such that the right-hand side is arbitrarily close to the left-hand side.

The result is obtained using Takemura [15, Theorem 1], which computes the best constant of some kind of a Sobolev inequality. In Section 4 , we give a concise proof for an $L^{p}$ extension of Theorem 1 of [15]. 


\section{Proof of Theorem 1}

Now, let us introduce the following $L^{p}$-type Sobolev inequality:

$$
\left(\sup _{-s \leq x \leq s}\left|u^{(m)}(x)\right|\right)^{p} \leq C \int_{-s}^{s}\left|u^{(M)}(x)\right|^{p} d x,
$$

where $u$ belongs to

$$
W(M, p):=\left\{u \mid u^{(M)} \in L^{p}(-s, s), u^{(i)}(-s)=0(i=0, \ldots, M-1)\right\},
$$

$1<p, m$ runs over the range $0 \leq m \leq M-1$, and $u^{(i)}$ is the $i$ th derivative of $u$ in a distributional sense. We denote by $C_{C F}(M, m, p)$ the best constant of the above Sobolev inequality (6). Here, we note that in [15], Takemura obtained the best constant for $p=2, m=0$ by constructing the Green function of the clamped-free boundary value problem. Although, for the proof of Theorem 1 , we simply need the value $C_{C F}(M, 0,2)$, we would like to compute $C_{C F}(M, m, p)$ for general $p$ and $m$ since the proof presented in Section 4 does not depend on special values of $p$ and $m$ and quite simplifies the proof of Theorem 1 of [15]. Now, we have the following propositions.

Proposition 1 The best constant of (6) is

$$
C_{C F}(M, m, p)=\frac{1}{\{(M-m-1) !\}^{p}}\left(\frac{(p-1)(2 s)^{\frac{p(M-m)-1}{p-1}}}{p(M-m)-1}\right)^{p-1},
$$

and it is attained by

$$
u_{*}(x)=\int_{-s}^{x} \frac{(x-t)^{M-1}}{(M-1) !} \cdot\left\{\frac{(s-t)^{M-1-m}}{(M-1-m) !}\right\}^{p-1} d t .
$$

Proposition 2 Suppose a $C^{2 M}[-s, s]$ solution of (4) with the clamped-free boundary condition exists, then it holds that

$$
\int_{-s}^{s} r^{+}(x) d x>\frac{1}{C_{C F}(M, 0,2)}
$$

Moreover, the estimate is sharp.

Proof of Theorem 1 Clearly, Theorem 1 is obtained from Propositions 1 and 2.

Thus, all we have to do is to show Propositions 1 and 2. Before proceeding with the proof of these propositions, we would like to show a corollary obtained from Proposition 1.

Corollary 1 Suppose a non-trivial solution $u$ of the non-linear equation

$$
(-1)^{M} u(x) u^{(2 M)}(x)-r(x)\left(u^{(m)}(x)\right)^{2}=0
$$

exists under the clamped-free boundary condition, where $m$ satisfies $(1 \leq m \leq M-1)$, then it holds

$$
\int_{-s}^{s} r^{+}(x) d x>\frac{\{(M-1-m) !\}^{2}(2(M-m)-1)}{(2 s)^{2(M-m)-1}} .
$$


The following are the examples of Theorem 1 and Corollary 1.

Example 1 The following example corresponds to the case $M=1$ and $r(x)=-6 /\left(-11 s^{2}+\right.$ $\left.2 s x+x^{2}\right)$ of (4) with the clamped-free boundary condition

$$
\left\{\begin{array}{l}
-u^{\prime \prime}(x)+\frac{6}{-11 s^{2}+2 s x+x^{2}} u(x)=0 \\
u(-s)=u^{\prime}(s)=0
\end{array}\right.
$$

It is easy to see that $u(x)=-(s+x)\left(11 s^{2}-2 s x-x^{2}\right)$ is the solution of the above equation. Moreover, it holds that

$$
\int_{-s}^{s} r^{+}(x) d x=\int_{-s}^{s}-\frac{6}{-11 s^{2}+2 s x+x^{2}} d x=\frac{\sqrt{3} \log (2+\sqrt{3})}{2 s}>\frac{1}{C_{C F}(1,0,2)}=\frac{1}{2 s} .
$$

Example 2 The following example corresponds to the case $M=2, m=1$ and $r(x)=$ $\left(3\left(17 s^{2}-6 s x+x^{2}\right)\right) /\left(2\left(7 s^{2}-4 s x+x^{2}\right)^{2}\right)$ of (10) with the clamped-free boundary condition

$$
\left\{\begin{array}{l}
u(x) u^{(4)}(x)-\frac{3\left(17 s^{2}-6 s x+x^{2}\right)}{2\left(7 s^{2}-4 s x+x^{2}\right)^{2}}\left(u^{\prime}(x)\right)^{2}=0 \\
u(-s)=u^{\prime}(-s)=u^{\prime \prime}(s)=u^{\prime \prime \prime}(s)=0
\end{array}\right.
$$

It is easy to see that $u(x)=(s+x)^{2}\left(17 s^{2}-6 s x+x^{2}\right)$ is the solution of the above equation. Moreover, it holds that

$$
\int_{-s}^{s} r^{+}(x) d x=\int_{-s}^{s} \frac{3\left(17 s^{2}-6 s x+x^{2}\right)}{2\left(7 s^{2}-4 s x+x^{2}\right)^{2}} d x=\frac{3+2 \sqrt{3} \pi}{12 s}>\frac{1}{C_{C F}(2,1,2)}=\frac{1}{2 s} .
$$

\section{Proof of Proposition 2}

Assuming Proposition 1, we first prove Proposition 2.

Proof of Proposition 2 Let $u$ be a solution of equation (4). Since $u$ satisfies the clamped-free boundary condition, multiplying (4) by $u$ and integrating it over $[-s, s]$, we have

$$
\begin{aligned}
\int_{-s}^{s}\left(u^{(M)}(x)\right)^{2} d x & =\int_{-s}^{s}(-1)^{M} u(x) u^{(2 M)}(x) d x=\int_{-s}^{s} r(x)(u(x))^{2} d x \\
& \leq\left(\sup _{-s \leq x \leq s}|u(x)|\right)^{2} \int_{-s}^{s} r^{+}(x) d x \\
& \leq C(M, 0,2) \int_{-s}^{s}\left(u^{(M)}(x)\right)^{2} d x \int_{-s}^{s} r^{+}(x) d x .
\end{aligned}
$$

Here, if $u^{(M)} \equiv 0$, then there exists $a_{i} \in \mathbb{R}(i=0, \ldots, M-1)$ such that $u(x)=\sum_{i=0}^{M-1} a_{i} x^{i}$. Since $u$ satisfies the clamped boundary condition at $x=-s$, we have $u \equiv 0$. This contradicts the assumption that $u$ is a non-trivial solution of (4). So, canceling $\left\|u^{(M)}\right\|_{2}^{2}$, we obtain

$$
\int_{-s}^{s} r^{+}(x) d x \geq \frac{1}{C_{C F}(M, 0,2)}
$$

Next, we show that the inequality (13) is strict. To see this, we note that in (12), if the equality holds for the first inequality, then $u$ is a constant. But, again from the clamped 
boundary condition at $x=-s$, we have $u \equiv 0$. Thus, the inequality is strict. Finally, we see (5) is sharp. For this purpose, let us define the functional

$$
J(\phi):=\frac{\int_{-s}^{s}\left|\phi^{(M)}\right|^{2} d x}{\int_{-s}^{s} \tilde{r}|\phi|^{2} d x} \quad(\phi \in W(M, 2), \phi \not \equiv 0)
$$

where $\tilde{r} \in C\left([-s, s], \mathbb{R}_{+}\right)$is defined later. By the standard argument of the variational method, $J$ has the minimizer $u \in W(M, 2)$ (see, for example, [16, Lemma 3]), i.e.,

$$
\lambda_{1}:=\min _{\phi \in W(M, 2), \phi \neq 0} J(\phi)=J(u) .
$$

Hence, it satisfies the Euler-Lagrange equation (as a classical solution by the regularity argument)

$$
(-1)^{M} u^{(2 M)}(x)=\lambda_{1} \tilde{r}(x) u(x) \quad(-s \leq x \leq s)
$$

Further, it holds that

$$
\begin{aligned}
\lambda_{1} & =\min _{\phi \in W(M, 2), \phi \neq 0} \frac{\int_{-s}^{s}\left|\phi^{(M)}\right|^{2} d x}{\int_{-s}^{s} \tilde{r}|\phi|^{2} d x}>\frac{\int_{-s}^{s}\left|\phi^{(M)}\right|^{2} d x}{\left(\sup _{-s \leq x \leq s}|\phi|\right)^{2} \int_{-s}^{s} \tilde{r} d x} \\
& \geq \frac{1}{C_{C F}(M, 0,2) \int_{-s}^{s} \tilde{r} d x} .
\end{aligned}
$$

Here, let us fix $\tilde{r}$ as

$$
\tilde{r}(x):= \begin{cases}\frac{x-s}{\delta}+1 & (s-\delta<x \leq s), \\ 0 & (-s \leq x \leq s-\delta) .\end{cases}
$$

For such $\tilde{r}$, let us substitute $\phi=u_{*}$ (of Proposition 1 ) into (15). It is easy to see that $u_{*}$ takes its maximum at $x=s$, hence by taking $\delta$ sufficiently small, we see that the right-hand side of (15) can be arbitrarily close to the left-hand side, i.e., for a small positive $\epsilon_{1}, \lambda_{1}$ can be written as

$$
\lambda_{1}=\frac{1}{C_{C F}(M, 0,2) \int_{-s}^{s} \tilde{r} d x}+\epsilon_{1}
$$

Putting $r=\lambda_{1} \tilde{r}$, we see from (14) a solution $u$ of

$$
(-1)^{M} u^{(2 M)}(x)=r(x) u(x) \quad(-s \leq x \leq s)
$$

exists, and from (16) $r$ satisfies

$$
\int_{-s}^{s} r(x) d x=\frac{1}{C(M, 0,2)}+\epsilon_{1} \int_{-s}^{s} \tilde{r} d x=\frac{1}{C(M, 0,2)}+\epsilon_{2}
$$

Hence, (5) is sharp. 
Proof of Corollary 1 Integrating equation (10), we have

$$
\begin{aligned}
\int_{-s}^{s}\left(u^{(M)}(x)\right)^{2} d x & =\int_{-s}^{s} r(x)\left(u^{(m)}(x)\right)^{2} d x \leq\left(\sup _{-s \leq x \leq s}\left|u^{(m)}(x)\right|\right)^{2} \int_{-s}^{s} r^{+}(x) d x \\
& \leq C(M, m, 2) \int_{-s}^{s}\left(u^{(M)}(x)\right)^{2} d x \int_{-s}^{s} r^{+}(x) d x .
\end{aligned}
$$

As in the proof of Proposition 2, by canceling $\left\|u^{(M)}\right\|_{2}^{2}$, we have

$$
\int_{-s}^{s} r^{+}(x) d x \leq \frac{1}{C_{C F}(M, m, 2)}
$$

Next, we show that the inequality (11) is strict. To see this, we note that in (19), the equality holds for the first inequality if and only if $u^{(m)}$ is a constant. Hence, from the clamped boundary condition at $x=-s$, we have $u^{(m)} \equiv 0$. So, there exists $a_{i} \in \mathbb{R}(i=0, \ldots, m-1)$ such that $u(x)=\sum_{i=0}^{m-1} a_{i} x^{i}$. But, again from the clamped boundary condition at $x=-s$, we have $u \equiv 0$. Thus, inequality (11) is strict.

\section{Proof of Proposition 1}

We prepare the following lemmas for the proof of Proposition 1.

Lemma 1 Suppose there exists a function $u_{*} \in W(M, p)$ which attains the best constant $C(M, n, p)$ of $(6)$, then it holds that

$$
\max _{-s \leq x \leq s}\left|u_{*}^{(m)}(x)\right|=\left|u_{*}^{(m)}(s)\right| .
$$

Proof Suppose it holds that

$$
\max _{-s \leq x \leq s}\left|u_{*}^{(m)}(x)\right|=\left|u_{*}^{(m)}(a)\right|
$$

where $a \not \equiv s$. Further, let us define

$$
\tilde{u}(x):= \begin{cases}0 & (-s \leq x \leq-a) \\ u_{*}(x+a-s) & (-a \leq x \leq s)\end{cases}
$$

Then it holds $\tilde{u} \in W(M, p)$ and

$$
\max _{-s \leq x \leq s}\left|\tilde{u}^{(m)}(x)\right|=\max _{-s \leq x \leq s}\left|u_{*}^{(m)}(x)\right|=\left|u_{*}^{(m)}(a)\right|
$$

and $\left\|\tilde{u}^{(M)}\right\|_{L^{p}(-s, s)}<\left\|u_{*}^{(M)}\right\|_{L^{p}(-s, s)}$. Hence,

$$
C(M, m, p)=\frac{\left(\max _{-s \leq x \leq s}\left|u_{*}^{(m)}(x)\right|\right)^{p}}{\left\|u_{*}^{(M)}\right\|_{L^{p}(-s, s)}}<\frac{\left(\max _{-s \leq x \leq s}\left|\tilde{u}^{(m)}(x)\right|\right)^{p}}{\left\|\tilde{u}^{(M)}\right\|_{L^{p}(-s, s)}}
$$

This contradicts the assumption that $C(M, m, p)$ is the best constant of (6). 
Lemma 2 Let

$$
H_{m}(x):=\frac{(-1)^{M-1-m}(x-s)^{M-1-m}}{(M-1-m) !},
$$

then for $u \in W(M, p)$ it holds that

$$
u^{(m)}(s)=\int_{-s}^{s} u^{(M)}(x) H_{m}(x) d x
$$

Proof Integrating by parts, we obtain the result.

Proof of Proposition 1 From Lemma 2, we see that if the function attains the best constant $C(M, m, p)$, it belongs to $W_{*}(M, m, p) \subset W(M, p)$ :

$$
W_{*}(M, m, p)=\left\{u \in W(M, p)\left|\max _{-s \leq x \leq s}\right| u^{(m)}(x)|=| u^{(m)}(s) \mid\right\} .
$$

Let $u \in W_{*}(M, m, p)$. Then applying Hölder's inequality to (20), we have

$$
\max _{-s \leq x \leq s}\left|u^{(m)}(x)\right|=\left|u^{(m)}(s)\right| \leq\left\|H_{m}\right\|_{L^{q}(-s, s)}\left\|u^{(M)}\right\|_{L^{p}(-s, s)},
$$

where $q$ satisfies $1 / p+1 / q=1$. Hence, if there exists the function $u_{*} \in W_{*}(M, m, p)$ which attains the equality of (21), it holds that $C(M, m, p)=\left\|H_{m}\right\|_{L^{q}(-s, s)}^{p}$. On the contrary, we see that the equality holds for (21) if and only if $u$ satisfies

$$
u^{(M)}(x)=\left(\operatorname{sgn} H_{m}(x)\right)\left|H_{m}(x)\right|^{q-1} .
$$

It is easy to see that

$$
u_{*}(x)=\int_{-s}^{x} \frac{(x-t)^{m-1}}{(M-1) !}\left(\operatorname{sgn} H_{m}(t)\right)\left|H_{m}(t)\right|^{q-1} d t=\int_{-s}^{x} \frac{(x-t)^{m-1}}{(M-1) !}\left\{\frac{(s-t)^{M-1-m}}{(M-1-m) !}\right\}^{\frac{1}{p-1}} d t
$$

satisfies (22) and belongs to $W_{*}(M, m, p)$. Thus, we have shown $C(M, m, p)=\left\|H_{m}\right\|_{L^{q}(-s, s)}^{p}$. Now, we compute $\left\|H_{m}\right\|_{L^{q}(-s, s)}^{p}$. It is

$$
\begin{aligned}
\left\|H_{m}\right\|_{L^{q}(-s, s)}^{p} & =\frac{1}{\{(M-1-m) !\}^{p}}\left\{\int_{-s}^{s}(s-x)^{q(M-1-m)} d x\right\}^{\frac{p}{q}} \\
& =\frac{1}{\{(M-1-m) !\}^{p}}\left\{\frac{(p-1)(2 s)^{\frac{p(M-m)-1}{p-1}}}{p(M-m)-1}\right\}^{p-1} .
\end{aligned}
$$

This completes the proof. 


\section{Author details}

${ }^{1}$ Department of Computer Science, National Defense Academy, 1-10-20 Hashirimizu, Yokosuka, 239-8686, Japan. ${ }^{2}$ Liberal Arts and Basic Sciences, College of Industrial Technology, Nihon University, 2-11-1 Shinei, Narashino, 275-8576, Japan. ${ }^{3}$ Graduate School of Mathematical Sciences, Faculty of Engineering Science, Osaka University, 1-3 Matikaneyamacho, Toyonaka, 560-8531, Japan. ${ }^{4} T o k y o$ Metropolitan College of Industrial Technology, 1-10-40 Higashi-ooi, Shinagawa, Tokyo, 140-0011, Japan.

Received: 29 May 2012 Accepted: 9 October 2012 Published: 23 October 2012

\section{References}

1. Brown, R, Hinton, D: Lyapunov inequalities and their applications. In: Rassias, TM (ed.) Surveys in Classical Inequalities. Kluwer Academic, Dordrecht (2000)

2. Tiryaki, A: Recent developments of Lyapunov-type inequalities. Adv. Dyn. Syst. Appl. 5, 231-248 (2010)

3. Levin, A: Distribution of the zeros of solutions of a linear differential equation. Sov. Math. Dokl. 5, 818-821 (1964)

4. Das, KM, Vatsala, AS: Green's function for $n-n$ boundary problem and an analogue of Hartman's result. J. Math. Anal. Appl. 51, 670-677 (1975)

5. Cheng, SS: Lyapunov inequality for system disconjugacy of even order differential equations. Tamkang J. Math. 22, 193-197 (1991)

6. Pachpatte, BG: On Lyapunov type inequalities for certain higher order differential equations. J. Math. Anal. Appl. 195, 527-536 (1995)

7. Parhi, N, Panigrash, S: On Lyapunov type inequality for third-order differential equations. J. Math. Anal. Appl. 233, 445-460 (1999)

8. Yang, X: On inequalities of Lyapunov type. Appl. Math. Comput. 134, 293-300 (2003)

9. Yang, X, Lo, K: Lyapunov-type inequality for a class of even-order differential equations. Appl. Math. Comput. 215 , 3884-3890 (2010)

10. Parhi, N, Panigrash, S: Lyapunov-type inequality for higher order differential equations. Math. Slovaca 52, 31-46 (2002)

11. Çakmak, D: Lyapunov type integral inequalities for certain higher order differential equations. Appl. Math. Comput. 216, 368-373 (2010)

12. He, X, Tang, X: Lyapunov-type inequalities for even order differential equations. Commun. Pure Appl. Anal. 11 465-473 (2012)

13. He, X, Zhang, Q: Lyapunov-type inequalities for a class of even-order differential equations. J. Inequal. Appl. 2012,5 (2012)

14. Watanabe, K, Yamagishi, H, Kametaka, Y: Riemann zeta function and Lyapunov-type inequalities for certain higher order differential equations. Appl. Math. Comput. 218, 3950-3953 (2011)

15. Takemura, $\mathrm{K}$ : The beat constant of Sobolev inequality corresponding to clamped-free boundary value problem for $(-1)^{M}(d / d x)^{2 M}$. Proc. Jpn. Acad. 85, 112-117 (2009)

16. Watanabe, K: Lyapunov type inequality for the equation including 1-dim p-Laplacian. Math. Inequal. Appl. 15 657-662 (2012)

doi:10.1186/1029-242X-2012-242

Cite this article as: Watanabe et al.: Lyapunov-type inequalities for $2 M$ th order equations under clamped-free

boundary conditions. Journal of Inequalities and Applications 2012 2012:242.

\section{Submit your manuscript to a SpringerOpen ${ }^{\circ}$ journal and benefit from:}

- Convenient online submission

- Rigorous peer review

Immediate publication on acceptance

- Open access: articles freely available online

- High visibility within the field

- Retaining the copyright to your article 